
\title{
$\begin{array}{ll}\text { Research Square } & \begin{array}{l}\text { Preprints are preliminary reports that have not undergone peer review. } \\ \text { They should not be considered conclusive, used to inform clinical practice, } \\ \text { or referenced by the media as validated information. }\end{array}\end{array}$
}

\section{The characteristics of airflow obstruction and its association with treatment response in the real world in different GOLD groups of COPD patients}

\author{
Qing Song \\ Second Xiangya Hospital https://orcid.org/0000-0002-9773-1517 \\ Yi-Yang Zhao \\ Central South University \\ Yu-Qin Zeng \\ Central South University \\ Cong Liu \\ Central South University \\ Wei Cheng \\ Central South University \\ Min-Hua Deng \\ Central South University \\ Xin Li \\ Hunan Prevention and Treatment Center for Occupational Diseases

\section{Li-Bing Ma} \\ Guilin Medical University Affiliated Hospital \\ Yan Chen \\ Central South University \\ Shan Cai \\ Central South University \\ Ping Chen ( $\nabla$ pingchen0731@csu.edu.cn ) \\ Central South University https://orcid.org/0000-0001-6707-8636
}

\section{Research}

Keywords: Chronic obstructive pulmonary disease, Global Initiative for Chronic Obstructive Lung Disease, Pulmonary function, Exacerbation, Mortality.

Posted Date: January 15th, 2021

DOI: https://doi.org/10.21203/rs.3.rs-145783/v1

License: (c) (i) This work is licensed under a Creative Commons Attribution 4.0 International License. Read Full License 


\section{Abstract}

Background: The Global Initiative for Chronic Obstructive Lung Disease (GOLD) 2017 separated spirometry from combined assessment to guide treatment. We aimed to analyze the characteristics of airflow obstruction and its association with treatment response in the real world in different GOLD groups of COPD patients.

Methods: For this prospective observational study, stable COPD outpatients were enrolled and divided into Groups A, B, C and D based on GOLD 2017, and followed-up for 18 months. Data on patient demographics, pulmonary function, COPD assessment test (CAT), Clinical COPD Questionnaire (CCQ), modified Medical Research Council (mMRC), exacerbations, mortality and treatments were collected to analyze the airflow obstruction and its association with treatment response.

Results: A total of 993 subjects were classified into Groups A ( $n=170,17.1 \%), B(n=360,36.3 \%), C(n=122,12.3 \%)$, and D $(n=341,34.3 \%)$. There were significant differences in mMRC, CAT, CCQ, exacerbations and hospitalizations among different groups $(P<0.001)$. Groups $B$ and $D$ had more airflow obstruction and ventilatory disorder than Groups $A$ and $C(P<0.05)$. In the same groups with different GOLD grades, the differences were mainly observed in BMI, CAT, CCQ and treatment with long-acting muscarinic antagonist (LAMA) and LAMA + long-acting $\beta 2$ agonist $(\mathrm{LABA})+$ inhaled corticosteroid $(\mathrm{ICS})(\mathrm{P}<0.05)$. After 18 months of follow-up, the number of exacerbations and hospitalizations were significantly different among different groups, with Group $D$ having the highest values. Also, there were more frequent exacerbators and a higher mortality rate in Group D. However, in the same groups with different GOLD grades, the mortality rates and number of exacerbations, hospitalizations and frequent exacerbators showed no differences.

Conclusion: There were significant differences in pulmonary function in different groups. However, different GOLD grades had no impact on future exacerbations and mortality rate in the same groups.

\section{Introduction}

Chronic obstructive pulmonary disease (COPD) is a common, preventable and treatable disease, characterized by respiratory symptoms and persistent airflow obstruction [1-2]. It is also the most serious chronic respiratory disease, and it becomes the fifth largest disease in the global economic burden and have the third largest fatality rate [3].

The goal of the Global Initiative for Chronic Obstructive Lung Disease (GOLD) program is to produce recommendations for the management of COPD based on the best scientific information available. The first edition of GOLD was released in 2001 and has been revised annually [4]. Until 2011, GOLD evaluated COPD patients based on symptoms, degree of airflow obstruction, exacerbation risk. According to combined COPD assessment, patients were divided into Groups A, B, C and D [5]. However, compared with spirometry classification, the combined COPD assessment cannot better predict mortality and other important clinical outcomes [6-8]. Therefore, the GOLD 2017 revised the assessment tool and separated spirometry. ABCD groups were then only determined based on COPD assessment test (CAT) or modified Medical Research Council (mMRC), and exacerbation history [9].

Pulmonary function is an important parameter for evaluating airflow obstruction and severity in COPD patients. A post-bronchodilator ratio of forced expiratory volume in one second (FEV1) to forced vital capacity (FVC) of $<0.70$ can be interpreted as continuous airflow obstruction. FEV1 is an important pulmonary function parameter that most of the clinical trial evidence about treatment efficacy in COPD is based on [1011]. Although GOLD 2017 separated spirometry from the old assessment tool, combining spirometry results with patient symptoms and exacerbation history remains vital for the diagnosis, treatment of COPD.

Kim et al. [12] found that there was no difference in the rate of decline in pulmonary function among different groups categorized by GOLD 2014 assessment tools. However, in the real world, the characteristics of airflow obstruction and its association with treatment response in different groups assessed according to GOLD 2017 is unclear.

\section{Methods}

\section{Study design and subjects}

This is a multicenter, prospective observational study, based on data collected as part of the Chronic Pulmonary Diseases Database setup by the Second Xiangya Hospital of Central South University (Registration number: ChiCTR-POC-17010431). Patients were enrolled from September 2017 to February 2019. All patients were followed-up for 18 months. According to criteria of GOLD 2017, COPD was confirmed when an FEV1/FVC ratio of $<0.70$ was obtained, following the inhalation of $400 \mu \mathrm{g}$ of salbutamol aerosol. Patients diagnosed with, or with a history of bronchiectasis, asthma, lung cancer or pneumonia were excluded from our study. This study was approved by the institutional ethics committee of the Second Xiangya Hospital of Central South University. All patients gave informed consent. 
The collected data included age, sex, education degree, body mass index (BMI), smoking history, biofuel and occupational exposure history, CAT, mMRC, Clinical COPD Questionnaire (CCQ), pulmonary function, exacerbations, hospitalizations and treatments. After 18 months of follow-up, data on exacerbations, hospitalizations and mortality were collected.

\section{Definition of exacerbation}

An exacerbation was defined as an acute worsening of respiratory symptoms that resulted in the need for additional therapy (including antibiotics, oral corticosteroids or require hospitalization) [13]. Frequent exacerbators were patients who suffered at least two exacerbations or one hospitalization per year or died [14].

\section{Classification of combined COPD assessment}

According to GOLD 2017, patients were assigned to four categories. Briefly, Group A, 0 to 1 exacerbation per year, no hospitalizations, CAT score $<10$ or mMRC score of 0 to 1 ; Group B, CAT score $\geq 10$ or mMRC score $\geq 2$; Group C, exacerbations $\geq 2$ or hospitalization $\geq 1$ per year, and a CAT score $<10$ or mMRC score of 0 to 1 ; Group D, CAT score $\geq 10$ or $\mathrm{mMRC}$ score $\geq 2$.

\section{Classification of GOLD grades}

GOLD grading was based on FEV1 \% predicted as follows: GOLD grade I, $\geq 80$ FEV1 \% predicted; GOLD grade II, 50-79 FEV1 \% predicted; GOLD grade III, 30-49 FEV1 \% predicted; GOLD grade IV, < 30 FEV1 \% predicted.

\section{Pulmonary function test}

Pulmonary function tests were measured by a spirometer (MasterScreen-Body/Diff, CareFusion, Germany). The following parameters were measured after a bronchodilator test: FEV1, FEV1 \% predicted, FVC, FVC \% predicted, FEV1/FVC, maximal expiratory flow (MEF) 25, MEF25 \% predicted, MEF75, MEF75\% predicted, peak expiratory flow (PEF), PEF \% predicted, and diastolic function test. The bronchodilator test was performed 20 minutes after inhaling $400 \mu \mathrm{g}$ of salbutamol aerosol. The level of ventilatory disorder before a bronchodilator test was determined using the FEV1 \% predicted value as follows: mild, $\geq 70$ FEV1 \% predicted; moderate, 60-69 FEV1 \% predicted; moderately-severe, 50-59 FEV1 \% predicted; severe, 35-49 FEV1 \% predicted and very severe, < 35 FEV1 \% predicted [15].

\section{Statistical analysis}

Statistical analyses were performed using SPSS 26 (IBM Corporation, Armonk, NY, USA). The data was expressed as the mean \pm standard deviation, or as the median and interquartile range. The Pearson's chi-squared test was used to analyze categorical variables. Comparisons of continuous variables were performed using independent-samples t-test or one-way analysis. The least significant difference (LSD) t-test was used for pairwise comparisons. The non-parametric test was used for non-normal distribution or uneven variance. $P$ values $<0.05$ were considered statistically significant.

\section{Results}

\section{Baseline demographic and clinical characteristics $(\mathrm{N}=993)$}

993 patients were analyzed in this study (Fig. 1). According to GOLD 2017, 170 (17.1\%), 360 (36.3\%), 122 (12.3\%) and 341 (34.3\%) patients were allocated to Groups A, B, C and D, respectively. The data on demographic and clinical characteristics are shown in the Table 1. The mean age of the enrolled patients from Groups $A$ to $D$ were $61.5 \pm 7.9,65.1 \pm 8.1,63.0 \pm 9.1$ and $66.8 \pm 8.0$ years and were significantly different $(P<$ 0.001). There were more current smokers in the Groups $A$ and $C(P<0.05)$. There were higher CAT and CCQ scores in Groups $B$ and $D(P<0.05)$ The proportion of LAMA was higher in Groups $A$ and $C$, while LAMA + LABA + ICS was higher in Groups $B$ and $D(P<0.05)$. There were higher numbers of exacerbations and hospitalizations in Groups $C$ and $D(P<0.05)$. The proportions of patients in $G$ roups $A, B, C$ and $D$ that suffered an exacerbation once per year were $13.5 \%, 13.1 \%, 50.8 \%$ and $26.7 \%$, respectively, while the proportion of patients suffering exacerbations at least twice per year in Groups C and D were 49.2\% and 3.3\%. The proportion of patients who were never hospitalized in Groups A, B, C and D were $100 \%, 100 \%, 28.7 \%$ and $31.4 \%$, respectively. However, the proportions of patients who were hospitalized at least once per year were $71.3 \%$ in Groups C and $68.6 \%$ in Group D. 
Table 1

The distribution of baseline demographic and clinical characteristics in different groups $(\mathrm{N}=993)$.

\begin{tabular}{|c|c|c|c|c|c|}
\hline Variables & $\begin{array}{l}\text { Group A } \\
(n=170)\end{array}$ & $\begin{array}{l}\text { Group B } \\
(n=360)\end{array}$ & $\begin{array}{l}\text { Group C } \\
(n=122)\end{array}$ & $\begin{array}{l}\text { Group D } \\
(n=341)\end{array}$ & $\begin{array}{l}\text { P- } \\
\text { value }\end{array}$ \\
\hline Age (years) & $61.5 \pm 7.9 * 8$ & $65.1 \pm 8.1^{9}$ & $63.0 \pm 9.1 \bullet$ & $66.8 \pm 8.0 \Delta$ & $<0.001$ \\
\hline Sex, n (\%) & & & & & 0.488 \\
\hline Male & 149 (87.6) & 320 (88.9) & 113 (92.6) & 299 (87.7) & \\
\hline Female & $21(12.4)$ & $40(11.1)$ & $9(7.4)$ & $42(12.3)$ & \\
\hline \multicolumn{6}{|l|}{ Education, n (\%) } \\
\hline Primary school & $59(34.7)$ *,\#, \& & $148(41.1)$ & $56(45.9) \bullet$ & $160(46.9) \boldsymbol{\Delta}$ & 0.050 \\
\hline Junior high school & $57(33.5)$ & $132(36.7)$ & 48 (39.3) & $120(35.2)$ & 0.751 \\
\hline High school & $36(21.2) * \#, \&$ & $63(17.5)^{9}$ & $11(9.0) \bullet$ & $47(13.8) \boldsymbol{\Lambda}$ & 0.021 \\
\hline University & $18(10.6){ }^{*}, \#, \&$ & $17(4.7)^{9}$ & $7(5.8) \bullet$ & $14(4.1)$ & 0.019 \\
\hline BMI $\left(\mathrm{kg} / \mathrm{m}^{2}\right)$ & $23.13 \pm 3.57$ & $22.70 \pm 3.87$ & $22.55 \pm 3.44$ & $22.24 \pm 3.71$ & 0.071 \\
\hline \multicolumn{6}{|l|}{$\begin{array}{l}\text { Smoke history, } \\
\mathrm{n}(\%)\end{array}$} \\
\hline Never-smoker & 30 (17.6) & $65(18.0)$ & 19 (15.6) & 69 (20.2) & 0.681 \\
\hline Ex-smoker & $43(25.3)$ & $119(33.1)$ & $36(29.5)$ & $126(37.0)$ & 0.054 \\
\hline Current-smoker & $97(57.1) *$ * \& & 176 (48.9) & $67(54.9) \bullet$ & $146(42.8) \boldsymbol{\Delta}$ & 0.010 \\
\hline $\begin{array}{l}\text { Smoke } \\
\text { (pack/year) } \\
\text { (Median, IQR) }\end{array}$ & $30(30)$ & $32(30)$ & $36.5(31.25)$ & $30(30)$ & 0.357 \\
\hline $\begin{array}{l}\text { Biofuel exposure, } \\
n(\%)\end{array}$ & & & & & $<0.001$ \\
\hline Yes & $\begin{array}{l}43(25.3) \\
*, \#, \&\end{array}$ & 147 (40.8) & $49(40.2)$ & $161(47.2)$ & \\
\hline No & $127(74.7) * \#, \&$ & $213(59.2)$ & 73 (59.8) & $180(52.8)$ & \\
\hline Occupational exposure, n (\%) & & & & & 0.471 \\
\hline Yes & $59(34.7)$ & $131(36.4)$ & $52(42.6)$ & $134(39.3)$ & \\
\hline No & $111(65.3)$ & $229(63.6)$ & $70(57.4)$ & $207(60.7)$ & \\
\hline $\begin{array}{l}\text { CAT } \\
(\text { Mean } \pm S D)\end{array}$ & $10.32 \pm 4.80 *$; \# \& & $16.61 \pm 5.09$ & $13.57 \pm 5.03$ & $19.21 \pm 5.78 \Delta$ & $<0.001$ \\
\hline $\begin{array}{l}\text { mMRC } \\
\text { (Median, IQR) }\end{array}$ & $1(0) *$, \& & $2(1)^{9}$ & $1(0) \bullet$ & $3(1)^{\wedge}$ & $<0.001$ \\
\hline $\begin{array}{l}\text { CCQ } \\
(\text { Mean } \pm S D)\end{array}$ & $15.85 \pm 5.89 * \#, \&$ & $22.80 \pm 5.71$ & $19.20 \pm 5.73 \bullet$ & $25.45 \pm 5.80 \Delta$ & $<0.001$ \\
\hline
\end{tabular}

Notes: * $P<0.05$ versus group $B, \# P<0.05$ versus group $C, \& P<0.05$ versus group $D, 9 P<0.05$ versus group $C, \$ P<0.05$ versus group $D$, $\Delta P<0.05$ versus group $B$. 


\begin{tabular}{|c|c|c|c|c|c|}
\hline Variables & $\begin{array}{l}\text { Group A } \\
(n=170)\end{array}$ & $\begin{array}{l}\text { Group B } \\
(n=360)\end{array}$ & $\begin{array}{l}\text { Group C } \\
(n=122)\end{array}$ & $\begin{array}{l}\text { Group D } \\
(n=341)\end{array}$ & $\begin{array}{l}\text { P- } \\
\text { value }\end{array}$ \\
\hline Any COPD medication & $157(92.4) *$; \#, \& & $351(97.5)^{9}$ & $115(94.3)$ & $328(96.2) \wedge$ & 0.039 \\
\hline LAMA & $87(51.2) * \&$ & $123(34.2){ }^{9}$ & $60(49.2) \bullet$ & $111(32.6)$ & $<0.001$ \\
\hline$L A B A+I C S$ & $12(7.1)$ & $33(9.2)$ & $10(8.2)$ & $18(5.3)$ & 0.257 \\
\hline LAMA + LABA & $1(0.6)$ & $2(0.6)$ & $0(0)$ & $4(1.2)$ & 0.716 \\
\hline $\begin{array}{l}\text { LAMA + LABA } \\
+ \text { ICS }\end{array}$ & $55(32.6) *$ * \& & $190(52.8)^{9}$ & $43(35.3) \bullet$ & $195(57.2)$ & $<0.001$ \\
\hline $\begin{array}{l}\text { Exacerbations } \\
\text { in the past year, } \\
\text { (Mean } \pm \text { SD) }\end{array}$ & $0.14 \pm 0.34$ \#, \& & $0.13 \pm 0.34$ & $2.46 \pm 2.55 \bullet$ & $3.46 \pm 4.10^{\Delta}$ & $<0.001$ \\
\hline $\begin{array}{l}\text { Exacerbations } \\
\text { in the past year, } \\
\mathrm{n}(\%)\end{array}$ & & & & & $<0.001$ \\
\hline 0 & $147(86.5)^{\#, \&}$ & $313(86.9) 9$ & $0(0)$ & $0(0) \wedge$ & \\
\hline 1 & $23(13.5) \#, \&$ & $47(13.1)^{9}$ & $62(50.8) \bullet$ & $91(26.7) \wedge$ & \\
\hline$\geq 2$ & $0(0) \#$ & $0(0)^{9}$ & $60(49.2) \bullet$ & $250(73.3) \wedge$ & \\
\hline $\begin{array}{l}\text { Hospitalizations in the past year } \\
\text { (Mean } \pm \text { SD) }\end{array}$ & $0 \#, \&$ & $0^{9}$ & $0.94 \pm 1.00$ & $1.38 \pm 1.49 \Delta$ & $<0.001$ \\
\hline $\begin{array}{l}\text { Hospitalizations in the past year, } \\
\text { n (\%) }\end{array}$ & & & & & $<0.001$ \\
\hline 0 & 170 (100) \#,\& & $360(100)^{9}$ & $35(28.7)$ & $107(31.4) \wedge$ & \\
\hline$\geq 1$ & $0(0) \#, \&$ & $0(0)^{9}$ & $87(71.3)$ & $234(68.6) \wedge$ & \\
\hline
\end{tabular}

\section{Characteristics of pulmonary function}

As shown in Table 2, there were significant differences across Groups A to D in FEV1, FEV1 \%predicted, FVC, FVC \%predicted, FEV1/FVC, MEF 25, MEF25 \%predicted, MEF75, MEF75 \%predicted, PEF and PEF \% predicted $(P<0.001)$. Groups B and D had a heavier airflow obstruction than Groups A and C. Also, the proportion of patients with mild and moderate ventilatory disorder was higher in Groups A and C, while the proportion of patients with very severe disorder was higher in Groups B and $D(P<0.05)$. Similarly, the proportion of GOLD grade I to IV patients was significantly different across Groups $A$ to $D(P<0.001)$. The proportion of GOLD grades I and II patients were higher in Group $A$, while GOLD grades III and IV were higher in Group $D(P<0.05)$. 
Table 2

Characteristics of pulmonary function in different groups $(\mathrm{N}=993)$.

\begin{tabular}{|c|c|c|c|c|c|}
\hline Variables & $\begin{array}{l}\text { Group A } \\
(n=170)\end{array}$ & $\begin{array}{l}\text { Group B } \\
(n=360)\end{array}$ & $\begin{array}{l}\text { Group C } \\
(n=122)\end{array}$ & $\begin{array}{l}\text { Group D } \\
(n=341)\end{array}$ & P-value \\
\hline FEV1 (L) & $1.71 \pm 0.57^{*}, \#, \&$ & $1.21 \pm 0.49$ ฯ & $1.56 \pm 0.64 \bullet$ & $1.08 \pm 0.57 \Delta$ & $<0.001$ \\
\hline $\begin{array}{l}\text { FEV1 } \\
\text { (\% predicted) }\end{array}$ & $65.74 \pm 18.44 * \#, \&$ & $49.72 \pm 18.78$ & $59.42 \pm 22.18$ & $45.71 \pm 18.20 \Delta$ & $<0.001$ \\
\hline FEV1/FVC & $54.53 \pm 10.51 * \#, \&$ & $44.67 \pm 12.53$ & $50.59 \pm 12.68 \bullet$ & $42.95 \pm 11.81 \Delta$ & $<0.001$ \\
\hline $\mathrm{FVC}(\mathrm{L})$ & $3.09 \pm 0.76 * 8$ & $2.70 \pm 0.65$ & $3.01 \pm 0.80 \bullet$ & $2.49 \pm 0.71 \Delta$ & $<0.001$ \\
\hline $\begin{array}{l}\text { FVC } \\
\text { (\% predicted) }\end{array}$ & $95.20 \pm 17.29 *, 8$ & $86.57 \pm 17.38$ & $91.25 \pm 18.45 \bullet$ & $82.35 \pm 18.77 \Delta$ & $<0.001$ \\
\hline MEF25 & $0.32 \pm 0.30 * \#, \&$ & $0.23 \pm 0.27$ & $0.30 \pm 0.20$ & $0.21 \pm 0.18 \Delta$ & $<0.001$ \\
\hline $\begin{array}{l}\text { MEF25 } \\
\text { (\% predicted) }\end{array}$ & $26.14 \pm 15.36 *$ * \& & $21.91 \pm 14.23$ & $24.32 \pm 14.94$ & $20.62 \pm 14.88$ & $<0.001$ \\
\hline MEF75 & $2.54 \pm 1.55^{*}$ *\#, \& & $1.46 \pm 1.129$ & $1.12 \pm 1.44$ & $1.21 \pm 0.99 \Delta$ & $<0.001$ \\
\hline $\begin{array}{l}\text { MEF75 } \\
\text { (\% predicted) }\end{array}$ & $38.46 \pm 23.48$ * $, \#, \&$ & $22.88 \pm 17.12$ & $32.01 \pm 21.89$ & $19.16 \pm 16.70 \Delta$ & $<0.001$ \\
\hline PEF (L/s) & $4.47 \pm 1.85^{*}, \#, \&$ & $3.25 \pm 1.32$ & $4.02 \pm 1.58$ & $2.90 \pm 1.33 \Delta$ & $<0.001$ \\
\hline PEF (\%predicted) & $62.12 \pm 23.83 * \#, \&$ & $46.88 \pm 18.77^{9}$ & $54.43 \pm 20.45 \bullet$ & $42.51 \pm 19.44 \Delta$ & $<0.001$ \\
\hline \multicolumn{6}{|l|}{$\begin{array}{l}\text { Ventilatory disorder, } \\
\mathrm{n}(\%)\end{array}$} \\
\hline Mild & $44(25.9) *$ * \& & 38 (10.6) & $27(22.1) \bullet$ & $24(7.0)$ & $<0.001$ \\
\hline Moderate & $44(25.9) *$ * \& & $41(11.4)^{9}$ & $23(18.9) \bullet$ & $25(7.4)$ & $<0.001$ \\
\hline Moderately-severe & $33(19.4)$ & $56(15.5)$ & $23(18.8)$ & $54(15.8)$ & 0.609 \\
\hline Severe & $38(22.4)$ & 100 (27.8) & $24(19.7)$ & $91(26.7)$ & 0.230 \\
\hline Very severe & $11(6.4)$ *,\#, \& & $125(34.7)$ ฯ & $25(20.5) \bullet$ & $147(43.1) \wedge$ & $<0.001$ \\
\hline $\begin{array}{l}\text { Diastolic function } \\
\text { Test, n (\%) }\end{array}$ & & & & & 0.121 \\
\hline Positive & $22(12.9)$ & $52(14.4)$ & $17(13.9)$ & $30(8.8)$ & \\
\hline Negative & $148(87.1)$ & 308 (85.6) & 105 (86.1) & 311 (91.2) & \\
\hline GOLD grade, $\mathrm{n}(\%)$ & & & & & \\
\hline I & $37(21.7) *$ * \& & $26(7.2) 9$ & $21(17.2) \bullet$ & $18(5.3)$ & $<0.001$ \\
\hline II & $104(61.2) * \#, \&$ & $148(41.1)$ & $57(46.7) \bullet$ & $105(30.8) \boldsymbol{\Delta}$ & $<0.001$ \\
\hline
\end{tabular}

Notes: * $P<0.05$ versus group $B, \# P<0.05$ versus group $C, \& P<0.05$ versus group $D, 9 P<0.05$ versus group $C, \$ P<0.05$ versus group $D$, $\Delta P<0.05$ versus group $B$. 


\begin{tabular}{|c|c|c|c|c|c|}
\hline Variables & $\begin{array}{l}\text { Group A } \\
(n=170)\end{array}$ & $\begin{array}{l}\text { Group B } \\
(n=360)\end{array}$ & $\begin{array}{l}\text { Group C } \\
(n=122)\end{array}$ & $\begin{array}{l}\text { Group D } \\
(n=341)\end{array}$ & P-value \\
\hline III & $26(15.3){ }^{*}, \#, \&$ & $130(36.1)$ & $33(27.1) \bullet$ & $149(43.7) \Delta$ & $<0.001$ \\
\hline IV & $3(1.8) * \#, \&$ & $56(15.6)^{9}$ & $11(9.0) \bullet$ & $69(20.2) \Delta$ & $<0.001$ \\
\hline
\end{tabular}


Table 3

Differences in baseline demographic and clinical characteristics for different GOLD grades in Groups $A, B, C$ and $D(N=993)$.

\begin{tabular}{|c|c|c|c|c|c|c|c|c|c|c|c|c|}
\hline \multirow[b]{2}{*}{ Variables } & \multicolumn{2}{|c|}{$\begin{array}{l}\text { Group A }(n= \\
170)\end{array}$} & \multirow{2}{*}{$\begin{array}{l}\text { P- } \\
\text { value }\end{array}$} & \multicolumn{2}{|c|}{ Group B $(n=360)$} & \multirow{2}{*}{$\begin{array}{l}\text { P- } \\
\text { value }\end{array}$} & \multicolumn{2}{|c|}{$\begin{array}{l}\text { Group } C(n= \\
122)\end{array}$} & \multirow{2}{*}{$\begin{array}{l}\text { P- } \\
\text { value }\end{array}$} & \multicolumn{2}{|c|}{ Group D $(n=341)$} & \multirow{2}{*}{$\begin{array}{l}\text { P- } \\
\text { value }\end{array}$} \\
\hline & GOLD & & & GOLD & & & GOLD & & & GOLD & & \\
\hline & I- II & III-IV & & I- II & III-IV & & I- II & III-IV & & I- II & III-IV & \\
\hline & $\begin{array}{l}(n= \\
141)\end{array}$ & $\begin{array}{l}(n= \\
29)\end{array}$ & & $\begin{array}{l}(n= \\
174)\end{array}$ & $\begin{array}{l}(n= \\
186)\end{array}$ & & $\begin{array}{l}(n= \\
78)\end{array}$ & $\begin{array}{l}(n= \\
44)\end{array}$ & & $(n=123)$ & $\begin{array}{l}(n= \\
218)\end{array}$ & \\
\hline Age (years) & $\begin{array}{l}61.0 \pm \\
7.6\end{array}$ & $\begin{array}{l}64.0 \pm \\
8.8\end{array}$ & 0.059 & $\begin{array}{l}66.4 \pm \\
8.2\end{array}$ & $\begin{array}{l}63.8 \pm \\
7.9\end{array}$ & 0.003 & $\begin{array}{l}62.9 \pm \\
9.3\end{array}$ & $\begin{array}{l}63.3 \pm \\
8.8\end{array}$ & 0.844 & $66.7 \pm 8.5$ & $\begin{array}{l}66.2 \\
\pm 7.7\end{array}$ & 0.108 \\
\hline Sex, n (\%) & & & 0.027 & & & 0.057 & & & 0.486 & & & 0.096 \\
\hline Male & $\begin{array}{l}120 \\
(85.1)\end{array}$ & $\begin{array}{l}29 \\
(100)\end{array}$ & & $\begin{array}{l}149 \\
(83.3)\end{array}$ & $\begin{array}{l}171 \\
(92.9)\end{array}$ & & $\begin{array}{l}71 \\
(91.0)\end{array}$ & $\begin{array}{l}42 \\
(95.4)\end{array}$ & & $103(83.7)$ & $\begin{array}{l}196 \\
(89.9)\end{array}$ & \\
\hline Female & $\begin{array}{l}21 \\
(14.9)\end{array}$ & $0(0)$ & & $\begin{array}{l}25 \\
(16.7)\end{array}$ & $15(7.5)$ & & $7(9.0)$ & $2(4.6)$ & & $20(16.3)$ & $\begin{array}{l}22 \\
(10.1)\end{array}$ & \\
\hline Education, n (\%) & & & 0.002 & & & 0.276 & & & 0.595 & & & 0.024 \\
\hline Primary school & $\begin{array}{l}45 \\
(31.9)\end{array}$ & $\begin{array}{l}14 \\
(47.3)\end{array}$ & & $\begin{array}{l}80 \\
(48.0)\end{array}$ & $\begin{array}{l}68 \\
(37.8)\end{array}$ & & $\begin{array}{l}36 \\
(46.2)\end{array}$ & $\begin{array}{l}20 \\
(45.5)\end{array}$ & & $71(57.7)$ & $\begin{array}{l}89 \\
(40.8)\end{array}$ & \\
\hline $\begin{array}{l}\text { Junior } \\
\text { high school }\end{array}$ & $\begin{array}{l}49 \\
(34.8)\end{array}$ & $\begin{array}{l}8 \\
(27.6)\end{array}$ & & $\begin{array}{l}57 \\
(33.3)\end{array}$ & $\begin{array}{l}75 \\
(40.4)\end{array}$ & & $\begin{array}{l}32 \\
(41.0)\end{array}$ & $\begin{array}{l}16 \\
(36.4)\end{array}$ & & $33(26.8)$ & $\begin{array}{l}87 \\
(39.9)\end{array}$ & \\
\hline High school & $\begin{array}{l}32 \\
(22.7)\end{array}$ & $\begin{array}{l}4 \\
(13.8)\end{array}$ & & $\begin{array}{l}28 \\
(13.3)\end{array}$ & $\begin{array}{l}35 \\
(17.9)\end{array}$ & & $5(6.4)$ & $\begin{array}{l}6 \\
(13.6)\end{array}$ & & $14(11.4)$ & $\begin{array}{l}33 \\
(15.2)\end{array}$ & \\
\hline University & $\begin{array}{l}15 \\
(10.6)\end{array}$ & $\begin{array}{l}3 \\
(10.3)\end{array}$ & & $9(5.4)$ & $8(3.9)$ & & $5(6.4)$ & $2(4.5)$ & & $5(4.1)$ & $\begin{array}{l}9 \\
(4.1)\end{array}$ & \\
\hline $\mathrm{BMI}\left(\mathrm{kg} / \mathrm{m}^{2}\right)$ & $\begin{array}{l}23.42 \\
\pm 3.50\end{array}$ & $\begin{array}{l}21.69 \\
\pm 3.58\end{array}$ & 0.017 & $\begin{array}{l}23.65 \\
\pm 3.50\end{array}$ & $\begin{array}{l}21.82 \\
\pm 3.84\end{array}$ & $\begin{array}{l}<.001 \\
0.00\end{array}$ & $\begin{array}{l}23.04 \\
\pm 3.54\end{array}$ & $\begin{array}{l}21.67 \\
\pm 3.10\end{array}$ & 0.034 & $\begin{array}{l}22.97 \pm \\
3.79\end{array}$ & $\begin{array}{l}21.65 \\
\pm 3.54\end{array}$ & 0.006 \\
\hline $\begin{array}{l}\text { Smoke history, } \\
\mathrm{n}(\%)\end{array}$ & & & 0.235 & & & 0.098 & & & 0.302 & & & 0.321 \\
\hline Never-smoker & $\begin{array}{l}28 \\
(19.9)\end{array}$ & $2(6.9)$ & & $\begin{array}{l}35 \\
(20.1)\end{array}$ & $\begin{array}{l}30 \\
(16.1)\end{array}$ & & $\begin{array}{l}15 \\
(19.2)\end{array}$ & $4(9.1)$ & & $27(22.0)$ & $\begin{array}{l}42 \\
(19.3)\end{array}$ & \\
\hline Ex-smoker & $\begin{array}{l}34 \\
(24.1)\end{array}$ & $\begin{array}{l}9 \\
(31.0)\end{array}$ & & $\begin{array}{l}48 \\
(27.6)\end{array}$ & $\begin{array}{l}71 \\
(38.2)\end{array}$ & & $\begin{array}{l}21 \\
(26.9)\end{array}$ & $\begin{array}{l}15 \\
(34.1)\end{array}$ & & 39 (31.7) & $\begin{array}{l}87 \\
(39.9)\end{array}$ & \\
\hline $\begin{array}{l}\text { Current- } \\
\text { smoker }\end{array}$ & $\begin{array}{l}79 \\
(56.0)\end{array}$ & $\begin{array}{l}18 \\
(62.1)\end{array}$ & & $\begin{array}{l}91 \\
(52.3)\end{array}$ & $\begin{array}{l}85 \\
(45.7)\end{array}$ & & $\begin{array}{l}42 \\
(53.9)\end{array}$ & $\begin{array}{l}25 \\
(56.8)\end{array}$ & & $57(46.3)$ & $\begin{array}{l}89 \\
(40.8)\end{array}$ & \\
\hline $\begin{array}{l}\text { Smoke } \\
\text { (pack/year) } \\
\text { (Median, IQR) }\end{array}$ & $\begin{array}{l}30 \\
(36.5)\end{array}$ & $\begin{array}{l}40 \\
(30)\end{array}$ & 0.248 & $\begin{array}{l}30 \\
(30)\end{array}$ & $\begin{array}{l}34.5 \\
(40)\end{array}$ & 0.376 & $\begin{array}{l}36.5 \\
(40)\end{array}$ & $\begin{array}{l}35.5 \\
(40)\end{array}$ & 0.236 & $30(30)$ & $\begin{array}{l}30 \\
(32)\end{array}$ & 0.322 \\
\hline $\begin{array}{l}\text { Biofuel exposure, } \\
\text { n (\%) }\end{array}$ & & & 0.875 & & & 0.676 & & & 0.201 & & & 0.181 \\
\hline Yes & $\begin{array}{l}36 \\
(25.5)\end{array}$ & $\begin{array}{l}7 \\
(24.1)\end{array}$ & & $\begin{array}{l}73 \\
(40.7)\end{array}$ & $\begin{array}{l}74 \\
(41.0)\end{array}$ & & $\begin{array}{l}28 \\
(35.9)\end{array}$ & $\begin{array}{l}21 \\
(47.7)\end{array}$ & & $64(52.0)$ & $\begin{array}{l}97 \\
(44.5)\end{array}$ & \\
\hline No & $\begin{array}{l}105 \\
(74.5)\end{array}$ & $\begin{array}{l}22 \\
(75.9)\end{array}$ & & $\begin{array}{l}101 \\
(59.3)\end{array}$ & $\begin{array}{l}112 \\
(59.0)\end{array}$ & & $\begin{array}{l}50 \\
(64.1)\end{array}$ & $\begin{array}{l}23 \\
(52.3)\end{array}$ & & $59(48.0)$ & $\begin{array}{l}121 \\
(55.5)\end{array}$ & \\
\hline $\begin{array}{l}\text { Occupational } \\
\text { exposure, n (\%) }\end{array}$ & & & 0.689 & & & 0.945 & & & 0.392 & & & 0.317 \\
\hline Yes & $\begin{array}{l}48 \\
(34.0)\end{array}$ & $\begin{array}{l}11 \\
(37.9)\end{array}$ & & $\begin{array}{l}63 \\
(35.3)\end{array}$ & $\begin{array}{l}68 \\
(37.8)\end{array}$ & & $\begin{array}{l}31 \\
(39.7)\end{array}$ & $\begin{array}{l}21 \\
(47.7)\end{array}$ & & $44(35.7)$ & $\begin{array}{l}90 \\
(41.3)\end{array}$ & \\
\hline No & $\begin{array}{l}93 \\
(66.0)\end{array}$ & $\begin{array}{l}18 \\
(62.1)\end{array}$ & & $\begin{array}{l}111 \\
(64.7)\end{array}$ & $\begin{array}{l}118 \\
(62.2)\end{array}$ & & $\begin{array}{l}47 \\
(60.3)\end{array}$ & $\begin{array}{l}23 \\
(52.3)\end{array}$ & & $79(64.3)$ & $\begin{array}{l}128 \\
(58.7)\end{array}$ & \\
\hline
\end{tabular}




\begin{tabular}{|c|c|c|c|c|c|c|c|c|c|c|c|c|}
\hline \multirow[b]{2}{*}{$\begin{array}{l}\text { CAT } \\
(\text { Mean } \pm \text { SD })\end{array}$} & \multicolumn{2}{|c|}{$\begin{array}{l}\text { Group } A(n= \\
170)\end{array}$} & \multirow{2}{*}{$\begin{array}{l}\text { P- } \\
0.006\end{array}$} & \multicolumn{2}{|c|}{ Group $B(n=360)$} & \multirow{2}{*}{$\begin{array}{l}P- \\
<.001\end{array}$} & \multicolumn{2}{|c|}{$\begin{array}{l}\text { Group } C(n= \\
122)\end{array}$} & \multirow{2}{*}{$\begin{array}{l}\text { P- } \\
0.034\end{array}$} & \multicolumn{2}{|c|}{ Group $D(n=341)$} & \multirow{2}{*}{$\begin{array}{l}\text { P- } \\
<.001\end{array}$} \\
\hline & $\begin{array}{l}9.87 \pm \\
4.67\end{array}$ & $\begin{array}{l}12.52 \\
\pm 4.92\end{array}$ & & $\begin{array}{l}15.49 \\
\pm 4.76\end{array}$ & $\begin{array}{l}17.66 \\
\pm 5.19\end{array}$ & & $\begin{array}{l}12.85 \\
\pm 4.86\end{array}$ & $\begin{array}{l}14.86 \\
\pm 5.19\end{array}$ & & $\begin{array}{l}17.63 \pm \\
5.32\end{array}$ & $\begin{array}{l}20.10 \\
\pm 5.85\end{array}$ & \\
\hline $\begin{array}{l}\text { mMRC } \\
\text { (Median, IQR) }\end{array}$ & $1(0)$ & $1(0)$ & 0.814 & $2(1)$ & $3(1)$ & <. 001 & $1(0)$ & $1(0)$ & 0.046 & $3(1)$ & $3(1)$ & $\dot{0} 001$ \\
\hline $\begin{array}{l}\text { CCQ } \\
(\text { Mean } \pm S D)\end{array}$ & $\begin{array}{l}15.18 \\
\pm 5.79\end{array}$ & $\begin{array}{l}19.10 \\
\pm 5.29\end{array}$ & 0.001 & $\begin{array}{l}21.60 \\
\pm 5.39\end{array}$ & $\begin{array}{l}23.93 \\
\pm 5.79\end{array}$ & $\hat{0} .001$ & $\begin{array}{l}18.15 \\
\pm 4.88\end{array}$ & $\begin{array}{l}21.05 \\
\pm 6.66\end{array}$ & 0.007 & $\begin{array}{l}23.80 \pm \\
5.64\end{array}$ & $\begin{array}{l}26.38 \\
\pm 5.69\end{array}$ & $\hat{0} .001$ \\
\hline \multicolumn{13}{|l|}{ Treatments, n(\%) } \\
\hline $\begin{array}{l}\text { Any COPD } \\
\text { medication }\end{array}$ & $\begin{array}{l}129 \\
(91.5)\end{array}$ & $\begin{array}{l}28 \\
(96.6)\end{array}$ & 0.700 & $\begin{array}{l}167 \\
(96.0)\end{array}$ & $\begin{array}{l}184 \\
(98.7)\end{array}$ & 0.095 & $\begin{array}{l}72 \\
(90.3)\end{array}$ & $\begin{array}{l}43 \\
(97.7)\end{array}$ & 0.420 & $117(95.1)$ & $\begin{array}{l}211 \\
(96.8)\end{array}$ & 0.557 \\
\hline LAMA & $\begin{array}{l}77 \\
(54.6)\end{array}$ & $\begin{array}{l}10 \\
(34.5)\end{array}$ & 0.048 & $\begin{array}{l}87 \\
(48.0)\end{array}$ & $\begin{array}{l}36 \\
(17.3)\end{array}$ & $\hat{0} .001$ & $\begin{array}{l}49 \\
(62.8)\end{array}$ & $\begin{array}{l}11 \\
(25.0)\end{array}$ & $\dot{0} 001$ & $69(56.1)$ & $\begin{array}{l}42 \\
(19.3)\end{array}$ & $\dot{0} .001$ \\
\hline $\mathrm{LABA}+\mathrm{ICS}$ & $\begin{array}{l}11 \\
(7.8)\end{array}$ & $1(3.5)$ & 0.693 & $\begin{array}{l}18 \\
(11.3)\end{array}$ & $\begin{array}{l}15 \\
(10.3)\end{array}$ & 0.454 & $7(9.0)$ & $3(6.8)$ & 0.677 & $7(5.7)$ & $\begin{array}{l}11 \\
(5.0)\end{array}$ & 0.798 \\
\hline $\begin{array}{l}\text { LAMA+ } \\
\text { LABA }\end{array}$ & $1(0.7)$ & 0 & 1.000 & 0 & $2(1.1)$ & 0.499 & 0 & 0 & - & 0 & $\begin{array}{l}4 \\
(1.8)\end{array}$ & 0.301 \\
\hline $\begin{array}{l}\text { LAMA+ } \\
\text { LABA + ICS }\end{array}$ & $\begin{array}{l}38 \\
(26.9)\end{array}$ & $\begin{array}{l}17 \\
(58.6)\end{array}$ & 0.001 & $\begin{array}{l}60 \\
(35.3)\end{array}$ & $\begin{array}{l}130 \\
(69.9)\end{array}$ & $\dot{0} .001$ & $\begin{array}{l}14 \\
(17.9)\end{array}$ & $\begin{array}{l}29 \\
(65.9)\end{array}$ & $\hat{0.001}$ & $40(32.5)$ & $\begin{array}{l}154 \\
(70.6)\end{array}$ & $\dot{0} 001$ \\
\hline $\begin{array}{l}\text { Exacerbations } \\
\text { in the past year } \\
\text { (Mean } \pm S D)\end{array}$ & $\begin{array}{l}0.13 \pm \\
0.34\end{array}$ & $\begin{array}{l}0.14 \pm \\
0.35\end{array}$ & 0.964 & $\begin{array}{l}0.13 \pm \\
0.34\end{array}$ & $\begin{array}{l}0.13 \pm \\
0.34\end{array}$ & 0.930 & $\begin{array}{l}2.32 \pm \\
2.28\end{array}$ & $\begin{array}{l}2.70 \pm \\
2.98\end{array}$ & 0.427 & $\begin{array}{l}3.39 \pm \\
3.84\end{array}$ & $\begin{array}{l}3.50 \\
\pm 4.24\end{array}$ & 0.820 \\
\hline $\begin{array}{l}\text { Hospitalizations } \\
\text { in the past year } \\
\text { (Mean } \pm S D \text { ) }\end{array}$ & 0 & 0 & - & 0 & 0 & - & $\begin{array}{l}0.92 \pm \\
1.07\end{array}$ & $\begin{array}{l}0.98 \pm \\
0.88\end{array}$ & 0.775 & $\begin{array}{l}1.34 \pm \\
1.42\end{array}$ & $\begin{array}{l}1.40 \\
\pm 1.53\end{array}$ & 0.712 \\
\hline
\end{tabular}

\section{Differences in demographic and clinical characteristics for different GOLD grades}

Each group was then divided into two subgroups included GOLD I-II and III-IV. In Group A, the proportions of patients in GOLD I-II and III-IV were $82.9 \%$ and $17.1 \%$, respectively, and there were significant differences between GOLD grade I-II and III-IV in sex, BMI, CAT, CCQ, treatment with LAMA and LAMA + LABA + ICS $(P<0.05)$. However, in Group B, the proportions of patients in GOLD I-II and III-IV were $48.3 \%$ and $51.7 \%$, respectively, and there were significant differences in age, BMI, CAT, MMRC, CCQ, treatment with LAMA and LAMA + LABA + ICS $(P<0.05)$. The proportions of patients in GOLD I-II and III-IV were $63.9 \%$ and $36.1 \%$ in group $\mathrm{C}$ and there were significant differences in BMI, CAT, $\mathrm{mMRC}, \mathrm{CCQ}$, treatment with LAMA and LAMA + LABA + ICS $(P<0.05)$. In contrast, in Group D, the proportions of patients in GOLD I-II and III-IV were $36.1 \%$ and $63.9 \%$, respectively, and there were significant differences in education degree, BMI, CAT, MMRC, CCQ, treatment with LAMA and LAMA + LABA + ICS between GOLD grade I-II and III-IV $(P<0.05)$

\section{Differences in future exacerbations and mortality in Groups A, B, C and D after 18 months of follow-up ( $=792)$}

After 18 months of follow-up, 792 patients were analyzed for future exacerbations and mortality. There were significant differences in numbers of exacerbations and hospitalizations among Groups $A, B, C$ and $D(P<0.001)$. The numbers of frequent exacerbators in $G$ roups $A, B$, $C$ and D were 15 (11.28\%), 56 (18.98\%), $24(23.30 \%)$ and 92 (35.25\%), respectively, while the percentage mortalities were significantly different among the four groups $(P<0.01)$ at $0.75 \%, 4.41 \%, 2.91 \%$ and $8.43 \%$, respectively. There were more frequent exacerbators and a higher mortality in group $\mathrm{D}$ (Table 4). 
Table 4

Exacerbations and mortality in Groups A, B, C and D after 18 months of follow-up $(\mathrm{N}=792)$.

\begin{tabular}{|c|c|c|c|c|c|}
\hline Variables & $\begin{array}{l}\text { Group A } \\
(n=133)\end{array}$ & $\begin{array}{l}\text { Group B } \\
(n=295)\end{array}$ & $\begin{array}{l}\text { Group C } \\
(n=103)\end{array}$ & $\begin{array}{l}\text { Group D } \\
(n=261)\end{array}$ & $\begin{array}{l}\text { P- } \\
\text { value }\end{array}$ \\
\hline $\begin{array}{l}\text { Exacerbations } \\
(\text { Mean } \pm \text { SD) }\end{array}$ & $0.18 \pm 0.46^{\&}$ & $0.33 \pm 0.78$ & $0.39 \pm 0.74 \bullet$ & $0.75 \pm 1.33 \Delta$ & $<0.001$ \\
\hline $\begin{array}{l}\text { Exacerbations, } \\
\mathrm{n}(\%)\end{array}$ & & & & & $<0.001$ \\
\hline 0 & $112(84.2) * \#, \&$ & $222(75.2)$ & $72(69.9) \bullet$ & $150(57.5) \wedge$ & \\
\hline 1 & $16(12.0)$ & $40(13.6)$ & $20(19.4)$ & $41(15.7)$ & \\
\hline$\geq 2$ & $4(3.0)^{\&}$ & $20(6.8)$ & $8(7.8) \bullet$ & $48(18.6) \boldsymbol{\Lambda}$ & \\
\hline Hospitalizations (Mean \pm SD) & $0.11 \pm 0.36^{\&}$ & $0.15 \pm 0.42$ & $0.25 \pm 0.59$ & $0.32 \pm 0.72 \wedge$ & 0.001 \\
\hline $\begin{array}{l}\text { Hospitalizations, } \\
\text { n (\%) }\end{array}$ & & & & & 0.001 \\
\hline 0 & 119 (89.4) *\#, \& & $247(83.7)^{9}$ & $80(77.7) \bullet$ & $183(70.1) \Delta$ & \\
\hline$\geq 1$ & $13(9.8)$ *\#, \& & $35(11.9)$ & $20(19.4) \bullet$ & $56(21.5)^{\wedge}$ & \\
\hline $\begin{array}{l}\text { Frequent } \\
\text { Exacerbators, } \\
\mathrm{n}(\%)\end{array}$ & $15(11.3) *$ * \#, \& & $56(19.0)$ & $24(23.3) \bullet$ & $92(35.3) \wedge$ & $<0.001$ \\
\hline Mortality, n (\%) & $1(0.8) *, \#, \&$ & $13(4.4)^{9}$ & $3(2.9) \bullet$ & $22(8.4)$ & 0.005 \\
\hline
\end{tabular}

As showed in Table 5, we divided each group into two subgroups (GOLD I-II and III-IV). In Groups A, B, C and D,there were no significant differences in the number of exacerbations or hospitalizations between GOLD I-II and III-IV patients after 18 months of follow-up. In addition, the proportion of patients with exacerbations $(0,1, \geq 2$ times/year) or hospitalizations $(0, \geq 1$ times/year) was not significantly different in the same group with different GOLD grades. The same trends could be seen in mortality rates and the proportion of frequent exacerbators in all groups. 
Table 5

Exacerbations and mortality for different GOLD grades in Groups A, B, C and D after 18 months of follow-up ( $N=792)$.

\begin{tabular}{|c|c|c|c|c|c|c|c|c|c|c|c|c|}
\hline \multirow[b]{2}{*}{ Variables } & \multicolumn{2}{|c|}{ Group A $(n=133)$} & \multirow{2}{*}{$\begin{array}{l}\text { P- } \\
\text { value }\end{array}$} & \multicolumn{2}{|c|}{ Group B $(n=295)$} & \multirow{2}{*}{$\begin{array}{l}\text { P- } \\
\text { value }\end{array}$} & \multicolumn{2}{|c|}{ Group C ( $n=103)$} & \multirow{2}{*}{$\begin{array}{l}\text { P- } \\
\text { value }\end{array}$} & \multicolumn{2}{|c|}{ Group D $(n=261)$} & \multirow{2}{*}{$\begin{array}{l}\text { P- } \\
\text { value }\end{array}$} \\
\hline & GOLD & & & GOLD & & & & & & & & \\
\hline & I- II & III-IV & & $\mathrm{H}$ & III-IV & & $\mathrm{H}-\mathrm{II}$ & III-IV & & H-II & III-IV & \\
\hline & $\begin{array}{l}(n= \\
109)\end{array}$ & $\begin{array}{l}(n= \\
24)\end{array}$ & & $\begin{array}{l}(n= \\
146)\end{array}$ & $\begin{array}{l}(n= \\
149)\end{array}$ & & $\begin{array}{l}(n= \\
63)\end{array}$ & $\begin{array}{l}(n= \\
40)\end{array}$ & & $\begin{array}{l}(n= \\
93)\end{array}$ & $\begin{array}{l}(n= \\
168)\end{array}$ & \\
\hline $\begin{array}{l}\text { Exacerbations } \\
\text { (Mean } \pm \text { SD) }\end{array}$ & $\begin{array}{l}0.19 \pm \\
0.46\end{array}$ & $\begin{array}{l}0.17 \pm \\
0.48\end{array}$ & 0.859 & $\begin{array}{l}0.31 \pm \\
0.74\end{array}$ & $\begin{array}{l}0.34 \pm \\
0.82\end{array}$ & 0.682 & $\begin{array}{l}0.32 \pm \\
0.60\end{array}$ & $\begin{array}{l}0.51 \pm \\
0.91\end{array}$ & 0.240 & $\begin{array}{l}0.64 \pm \\
1.21\end{array}$ & $\begin{array}{l}0.82 \pm \\
1.40\end{array}$ & 0.314 \\
\hline $\begin{array}{l}\text { Exacerbations, } \mathrm{n} \\
(\%)\end{array}$ & & & 0.671 & & & 0.935 & & & 0.582 & & & 0.264 \\
\hline 0 & $\begin{array}{l}91 \\
(84.3)\end{array}$ & $\begin{array}{l}21 \\
(87.5)\end{array}$ & & $\begin{array}{l}108 \\
(78.8)\end{array}$ & $\begin{array}{l}114 \\
(78.6)\end{array}$ & & $\begin{array}{l}46 \\
(75.4)\end{array}$ & $\begin{array}{l}26 \\
(66.7)\end{array}$ & & $\begin{array}{l}60 \\
(67.4)\end{array}$ & $\begin{array}{l}90 \\
(60.0)\end{array}$ & \\
\hline 1 & $\begin{array}{l}14 \\
(13.0)\end{array}$ & $2(8.3)$ & & $\begin{array}{l}20 \\
(14.6)\end{array}$ & $\begin{array}{l}20 \\
(13.8)\end{array}$ & & $\begin{array}{l}11 \\
(18.0)\end{array}$ & $\begin{array}{l}9 \\
(23.1)\end{array}$ & & $\begin{array}{l}16 \\
(18.0)\end{array}$ & $\begin{array}{l}25 \\
(16.7)\end{array}$ & \\
\hline 2 & $3(2.7)$ & $1(4.2)$ & & $9(6.6)$ & $\begin{array}{l}11 \\
(7.6)\end{array}$ & & $4(6.6)$ & $\begin{array}{l}4 \\
(10.2)\end{array}$ & & $\begin{array}{l}13 \\
(14.6)\end{array}$ & $\begin{array}{l}35 \\
(23.3)\end{array}$ & \\
\hline $\begin{array}{l}\text { Hospitalizations, } \\
\text { (Mean } \pm \text { SD) }\end{array}$ & $\begin{array}{l}0.13 \pm \\
0.39\end{array}$ & $\begin{array}{l}0.04 \pm \\
0.20\end{array}$ & 0.121 & $\begin{array}{l}0.15 \pm \\
0.39\end{array}$ & $\begin{array}{l}0.15 \pm \\
0.45\end{array}$ & 0.910 & $\begin{array}{l}0.20 \pm \\
0.44\end{array}$ & $\begin{array}{l}0.33 \pm \\
0.77\end{array}$ & 0.332 & $\begin{array}{l}0.31 \pm \\
0.81\end{array}$ & $\begin{array}{l}0.32 \pm \\
0.67\end{array}$ & 0.956 \\
\hline $\begin{array}{l}\text { Hospitalizations, } \\
\text { n (\%) }\end{array}$ & & & 0.461 & & & 0.719 & & & 0.539 & & & 0.367 \\
\hline 0 & $\begin{array}{l}96 \\
(88.89)\end{array}$ & $\begin{array}{l}23 \\
(95.83)\end{array}$ & & $\begin{array}{l}119 \\
(86.86)\end{array}$ & $\begin{array}{l}128 \\
(88.28)\end{array}$ & & $\begin{array}{l}50 \\
(81.97)\end{array}$ & $\begin{array}{l}30 \\
(76.92)\end{array}$ & & $\begin{array}{l}71 \\
(79.78)\end{array}$ & $\begin{array}{l}112 \\
(74.67)\end{array}$ & \\
\hline$\geq 1$ & $\begin{array}{l}12 \\
(11.11)\end{array}$ & $\begin{array}{l}1 \\
(4.17)\end{array}$ & & 18 (18) & $\begin{array}{l}17 \\
(11.72)\end{array}$ & & $\begin{array}{l}11 \\
(18.03)\end{array}$ & $\begin{array}{l}9 \\
(23.08)\end{array}$ & & $\begin{array}{l}18 \\
(20.22)\end{array}$ & $\begin{array}{l}38 \\
(25.33)\end{array}$ & \\
\hline $\begin{array}{l}\text { Frequent } \\
\text { exacerbator, } \\
\text { n (\%) }\end{array}$ & $\begin{array}{l}14 \\
(12.84)\end{array}$ & $\begin{array}{l}1 \\
(4.17)\end{array}$ & 0.305 & $\begin{array}{l}30 \\
(20.55)\end{array}$ & $\begin{array}{l}26 \\
(17.45)\end{array}$ & 0.497 & $\begin{array}{l}14 \\
(22.22)\end{array}$ & $\begin{array}{l}10 \\
(25.00)\end{array}$ & 0.745 & $\begin{array}{l}27 \\
(29.03)\end{array}$ & $\begin{array}{l}65 \\
(38.69)\end{array}$ & 0.118 \\
\hline Mortality, n (\%) & $1(0.9)$ & $0(0)$ & 0.638 & $\begin{array}{l}9 \\
(6.16)\end{array}$ & $\begin{array}{l}4 \\
(2.68)\end{array}$ & 0.145 & $\begin{array}{l}2 \\
(3.17)\end{array}$ & $\begin{array}{l}1 \\
(2.50)\end{array}$ & 0.843 & $\begin{array}{l}4 \\
(4.30)\end{array}$ & $\begin{array}{l}18 \\
(10.71)\end{array}$ & 0.074 \\
\hline
\end{tabular}

\section{Discussion}

In this study, stable COPD outpatients were divided into Groups A, B, C, and D based on GOLD 2017 to analyze the clinical features, the characteristics of airflow obstruction and its association with treatment response in the real world. We found that patients were older in Groups B and D. A similar result was observed in Oishi et al. [16] Smoking is a major environmental risk factors for COPD [17]. In this study, we found that compared with Group D, there were more current smoker in Groups A, B and C. Perhaps patients in Group D experienced more symptoms, which made more patients stop smoking. The number of female patients in this study was small. This may be because smoking was the main risk factor for COPD, but there were relatively few female smoking patients in China [18-19]. Biofuel exposure is another risk factor for the development of COPD that particularly affects women in developing countries [20-21]. Our research results also confirmed that Groups B to D had a higher biofuel exposure rate than Group A.

Since GOLD 2017 revised the assessment tool, the characteristics of airflow obstruction in Groups A, B, C and D were unclear. In this study, we found that there were the highest FEV1, FEV1 \% predicted, FVC, FVC \% predicted, FEV1/FVC, MEF 25, MEF25\% predicted, MEF75, MEF75 \% predicted, PEF and PEF \% predicted values in Group A. Also, there were more patients of mild and moderate ventilatory disorder and patients in GOLD grade I and II in Groups A. When compared with Group A, the airflow obstruction of Group C was higher and there were more patients of GOLD grade II and III in this group. The FEV1, FEV1 \% predicted, FVC, FVC \% predicted, FEV1/FVC, MEF 25, MEF25 \% predicted, MEF75, MEF75 $\%$ predicted, PEF and PEF \% predicted values were lowest in group D. In addition, in Group D, there were more patients with severe and very severe ventilatory disorder and patients in GOLD grade III and IV. In Group B, we could see that the airflow obstruction was lower than Group D, while higher than that in Group C. Furthermore, there were more patients with severe and very severe ventilatory disorder, and GOLD grade II and III in Groups B. Further research found that GOLD grade I and II patients were concentrated in Groups A and C, while GOLD III and IV 
patients were concentrated in Groups B and D. This is consistent with the results of Cabrera et al. [22] However, the proportion of GOLD IV patients in Groups A was relatively small. This was associated with less symptoms and a lower risk in group A patients.

In the GOLD 2011 guidelines, GOLD classification of airflow obstruction was used to guide combined COPD assessment. Briefly, GOLD I-II categories indicated low risk, while GOLD III-IV indicated high risk [23]. Therefore, we divided Groups A, B, C and D into two subgroups, one for GOLD I-II patients, and one for GOLD III-IV patients. The purpose of this was to analyze the differences in demographics and clinical characteristics. The results showed that patient with GOLD III-IV had lower BMI and proportion of LAMA, but higher CAT, CCQ and proportions of LAMA + LABA + ICS. This result implied that different GOLD grades had an impact on symptoms scores and treatments in the same groups.

Since GOLD 2017 removed spirometry, there was no research of its association with treatment response in real world in different groups. Therefore, we analyzed the future exacerbations and mortality in Groups $A, B, C$ and $D$, and in the same groups with different GOLD grades, after 18 months of follow-up. The period of 18 months was chosen because one-year follow-up times were not well reflective of future exacerbations in COPD patients [24]. The result showed that the exacerbations and hospitalizations were significantly different among different groups. The proportion of frequent exacerbators and mortality rates showed the same results. Further analysis found that there were more exacerbations and hospitalizations in Group D, along with a higher mortality rate. However, it was noted that the mortality rate was relatively low in this study because the patients were only followed-up for 18 months. Furthermore, we conducted analysis of the GOLD grade subgroups of Groups A, B, C and D. The results were surprising in that there were no differences in numbers of exacerbations or hospitalizations in all groups after 18 months of follow-up. Also, the mortality rates and proportions of frequent exacerbators were not significantly different. This result implied that GOLD classification of airway obstruction had no impact on the ABCD grouping in terms of future exacerbations and mortality. In other words, as described in the GOLD 2017 guidelines, combined COPD assessment should separate spirometry from the "ABCD" grouping [9].

There was still limitation in this study. The number of enrolled patients in Groups A and C was small. It may be that patients in Groups A and C have few symptoms, and typically in China, people attend hospital only once their symptoms are more severe.

\section{Conclusions}

In summary, our study revealed that there were significant differences in pulmonary function across Groups A to D, and that Groups B and D had more airflow obstruction. Also, there were significant differences in the number of exacerbations and the mortality rates among different groups after 18 months of follow-up. However, further analyzed showed there were no significant differences in exacerbations, hospitalizations and mortality rates in the same groups with different GOLD grades. In other words, GOLD classification of airway obstruction had no impact on future exacerbations and mortality rates in Groups A, B, C and D.

\section{Abbreviations List}

BMI, Body Mass Index

COPD, Chronic Obstructive Pulmonary Disease;

CAT, COPD Assessment Test

CCQ, Clinical COPD Questionnaire

FEV1, Forced Expiratory Volume in one second

FVC, Forced Vital Capacity

GOLD, Global Initiative for Chronic Obstructive Lung Disease

ICS, Inhaled Corticosteroid

LAMA, Long-Acting Muscarinic Antagonist

LABA, Long-Acting $\beta 2$-Agonist

MEF, Maximal Expiratory Flow

mMRC, Modified Medical Research Council

PTF, Pulmonary Function Tests

Page 12/15 
PEF, Peak Expiratory Flow.

\section{Declarations}

\section{Ethics approval and consent to participate}

This study was approved by the institutional ethics committee of the Second Xiangya Hospital of Central South University. All patients gave informed consent.

\section{Consent for publication}

Not applicable

\section{Availability of data and materials}

All publications discussed in the manuscript are available from the corresponding author on request.

\section{Competing interests}

The authors declare that they have no competing interests.

\section{Funding}

This work was supported by grants from the National Natural Science Foundation of China (NSFC, Grants 81770046 to Prof Ping Chen),

\section{Author's contributions}

Ping Chen, Yan Chen and Shan Cai were the guarantor and took responsibility for the content of this manuscript. Qing Song contributed toward data analysis, wrote this manuscript, drew the figures and tables. Yi-Yang Zhao, Yu-Qin Zeng, Wei Cheng, Cong Liu, Min-Hua Deng, Xin Li and Li-Bing Ma contributed to data collection and analysis.

\section{Acknowledgments}

The authors would like to thank the staff of the hospitals for their cooperation in collecting the study data.

\section{References}

1. Lareau SC, Fahy B, Meek P, Wang A. Chronic Obstructive Pulmonary Disease (COPD). Am J Respir Crit Care Med. 2019;199(1):P1-P2.

2. Smith MC, Wrobel JP. Epidemiology and clinical impact of major comorbidities in patients with COPD. Int J Chron Obstruct Pulmon Dis. 2014;9:871-88.

3. Raherison C, Girodet PO. Epidemiology of COPD. Eur Respir Rev. 2009;18(114):213-21.

4. Lee SJ, Yun SS, Ju S, You JW, Cho YJ, Jeong YY, et al. Validity of the GOLD 2017 classification in the prediction of mortality and respiratory hospitalization in patients with chronic obstructive pulmonary disease. Int J Chron Obstruct Pulmon Dis. 2019;14:911-919.

5. Vestbo J, Hurd SS, Agustí AG, Jones PW, Vogelmeier C, Anzueto A, et al. Global strategy for the diagnosis, management, and prevention of chronic obstructive pulmonary disease: GOLD executive summary. Am J Respir Crit Care Med. 2013;187(4):347-365.

6. Soriano JB, Lamprecht B, Ramírez AS, Martinez-Camblor P, Kaiser B, Alfageme I, et al. Mortality prediction in chronic obstructive pulmonary disease comparing the GOLD 2007 and 2011 staging systems: a pooled analysis of Individual patient data. Lancet Respir Med. 2015; 3(6):443-50.

7. Goossens LM, Leimer I, Metzdorf N, Becker K, Rutten-van Molken MP. Does the 2013 GOLD classification improve the ability to predict lung function decline, exacerbations and mortality: a post-hoc analysis of the 4-year UPLIFT trial. BMC Pulm Med. 2014;14:163.

8. Leivseth L, Brumpton BM, Nilsen TI, Mai XM, Johnsen R, Langhammer A. GOLD classifications and mortality in chronic obstructive pulmonary disease: the HUNT Study, Norway. Thorax. 2013;68(10):914-21.

9. Vogelmeier CF, Criner GJ, Martinez FJ, Anzueto A, Barnes PJ, Bourbeau J, et al. Global strategy for the diagnosis, management, and prevention of chronic obstructive lung disease 2017 report. GOLD executive summary. Am J Respir Crit Care Med. 2017;195(5):557-582.

10. Pauwels RA, Lofdahl CG, Laitinen LA, Schouten JP, Postma DS, Pride NB, et al. Long-term treatment with inhaled budesonide in persons with mild chronic obstructive pulmonary disease who continue smoking. European Respiratory Society Study on Chronic Obstructive Pulmonary Disease. N Engl J Med.1999;340(25):1948-53. 
11. Burge PS, Calverley PM, Jones PW, Spencer S, Anderson JA, Maslen TK. Randomised, double blind, placebo controlled study of fluticasone propionate in patients with moderate to severe chronic obstructive pulmonary disease:the ISOLDE trial. BMJ 2000;320(7245):1297-303.

12. Kim J, Yoon HI, Oh YM, Lim SY, Lee JH, Kim TH, et al. Lung function decline rates according to GOLD group in patients with chronic obstructive pulmonary disease. Int J Chron Obstruct Pulmon Dis. 2015;10:1819-27.

13. Rodriguez-Roisin R. Toward a consensus definition for COPD exacerbations. Chest 2000; 117: 398S-401S.

14. Hurst JR, Vestbo J, Anzueto A, Locantore N, Müllerova H, Tal-Singer R, et al. Susceptibility to exacerbation in chronic obstructive pulmonary disease. N Engl J Med. 2010;363(12):1128-38.

15. Pellegrino R, Viegi G, Brusasco V, Crapo RO, Burgos F, Casaburi R, et al. Interpretative strategies for lung function tests. Eur Respir J 2005; 26: $948-968$.

16. Oishi K, Hirano T, Hamada K, Uehara S, Suetake R, Yamaji Y, et al. Characteristics of 2017 GOLD COPD group A: a multicenter crosssectional CAP study in Japan. Int J Chron Obstruct Pulmon Dis. 2018;13:3901-3907.

17. Olloquequi J, Jaime S, Parra V, Cornejo-Córdova E, Valdivia G, Agustí À, et al. Comparative analysis of COPD associated with tobacco smoking, biomass smoke exposure or both. Respir Res. 2018;19(1):13.

18. Wang C, Xu J, Yang L, Xu Y, Zhang X, Bai C, et al. Prevalence and risk factors of chronic obstructive pulmonary disease in China (the China Pulmonary Health [CPH] study): a national cross-sectional study. 2018;391(10131):1706-1717.

19. Fang L, Gao P, Bao H, Tang X, Wang B, Feng Y, et al. Chronic obstructive pulmonary disease in China: a nationwide prevalence study. Lancet Respir Med. 2018;6(6):421-430.

20. Po JY, FitzGerald JM, Carlsten C. Respiratory disease associated with solid biomass fuel exposure in rural women and children: systematic review and meta-analysis. Thorax. 2011 Mar;66(3):232-9.

21. Pathak U, Gupta NC, Suri JC. Risk of COPD due to indoor air pollution from biomass cooking fuel: a systematic review and meta-analysis. Int J Environ Health Res. 2020 Feb;30(1):75-88.

22. Cabrera López C, Casanova Macario C, Marín Trigo JM, de-Torres JP, Sicilia Torres R, González JM, et al. Comparison of the 2017 and 2015 Global Initiative for Chronic Obstructive Lung Disease Reports. Impact on Grouping and Outcomes. Am J Respir Crit Care Med. 2018;197(4):463-469.

23. Agusti A, Edwards LD, Celli B, Macnee W, Calverley PM, Müllerova H, et al. Characteristics, stability and outcomes of the 2011 GOLD COPD groups in the ECLIPSE cohort. Eur Respir J.2013;42(3):636-46.

24. Sadatsafavi M, McCormack J, Petkau J, Lynd LD, Lee TY, Sin DD. Should the number of acute exacerbations in the previous year be used to guide treatments in COPD? Eur Respir J. 2020;2002122.

\section{Figures}




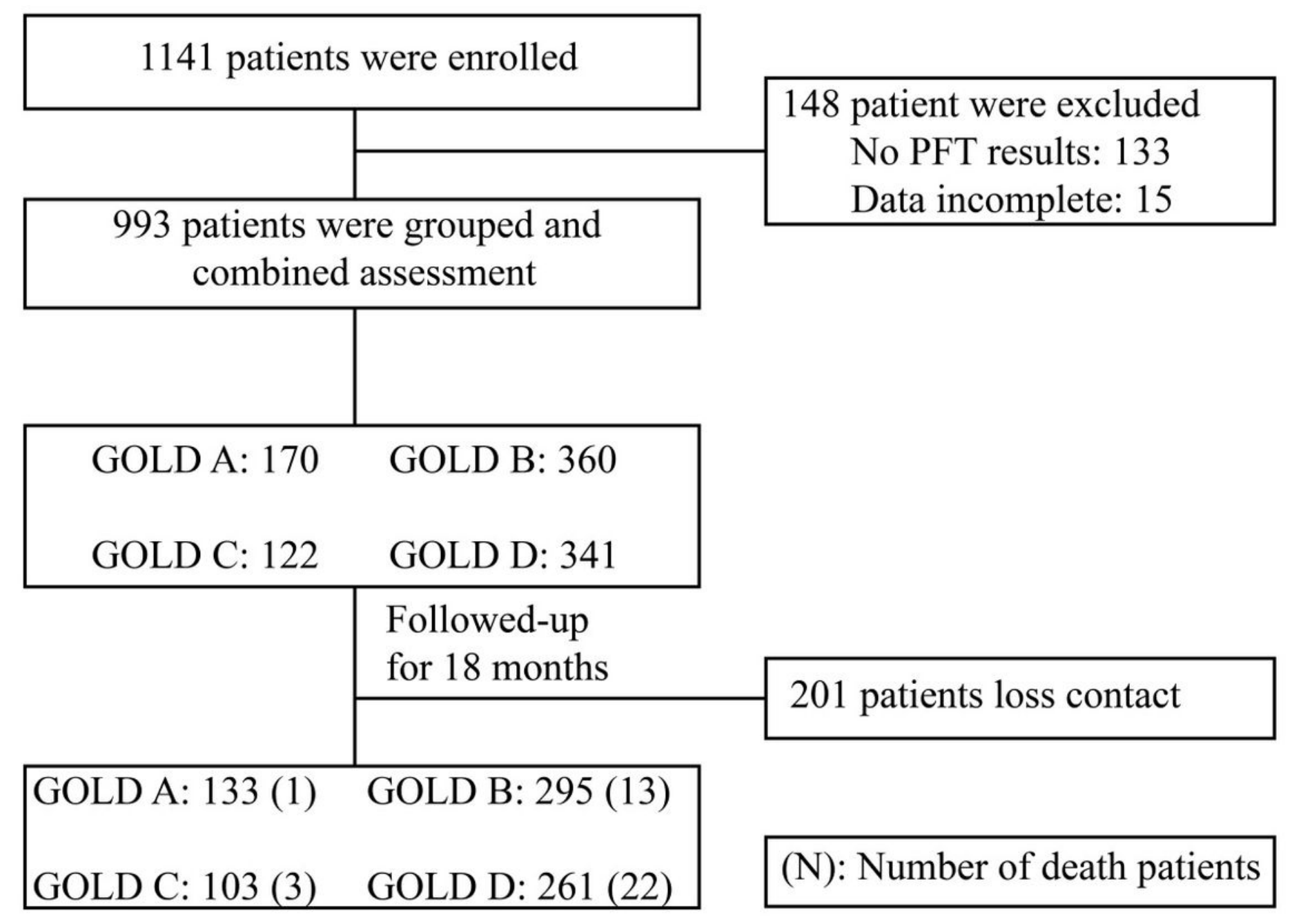

\section{Figure 1}

Flow chart of study inclusion. Note: Groups A to D were categories according to GOLD 2017. Abbreviations: GOLD, Global Initiative for Chronic Obstructive Lung Disease; PFT, Pulmonary Function Test. 